\title{
SOUS L'éGIDE DE LA CHRONIQUE
}

\section{POSTISCRIPTUM: CORRESPONDANCES}

\section{PHILOSOPHIQUES ENTRE LES DEUX RIVES DU RHIN}

A propos de Lettres d'Allemagne: Victor Cousin et les hegeliens, lettres rassemblees par Michel Espagne et Michael Werner avec la collaboration de Franģoise Lagnier.'

pout THetia BIRKENHAUER, Barbara WAHLSTER, UIric J. SCHNEIDER, et pont Jacgues POULAN

"P.S. L'argument du Gorgias que Vous m'aviez annonce, ne m'est pas parvenu." S'est-il perdu en route, ou n'a-t-il point été envoyé? Mais au fait, quelle importance? Pourtant, Friedrich Wilhem Carové, juriste et historien du catholicisme, compromis dans la défense des idées libérales, ancien répétiteur de Hegel à ses débuts à l'Université de Berlin et présentement fort occupé à se faire l'écho de la nouvelle philosophie française ${ }^{2}$ en Allemagne, y revient dans un autre post-scriptum à une lettre adressée de Francfort à son correspondant parisien, presque un an plus tard, le 11 avril 1828: "P.S. Je vous serais bien obligé si vous vouliez me communiquer vos arguments de Platon." Et le 22 septembre 1828:

"J'attends vos fragmens avec une bien grande impatience. Vos études platoniques vous ont placé au centre de la philosophie grecque; vous pouvez y avoir découvert beaucoup de choses qui n'ont pas été aperçues par vous autres. Je vous serai doublement obligé si vous vouliez bien y joindre Vos argumens de Platon. Vous voyez que je n'oublie pas vos promesses; mais je n'oublie pas non plus les miennes. Cest pour celà que je vous offre ci-joint mon dernier

${ }^{1}$ (Tusson, Charente: Du Lerot, Editeur, 1990).

2 F.V. Carove. Der Saint-Simonismus und die neuere franzosische Philosophie. (Leipzig: Hinrichsche Buchhandlung, 1831). 
ouvrage, bien que je crois qu'il ne vous intéressera pas assez pour le lire d'un bout a lautre."

Tout s'éclaire. Le dialogue entre les deux rives du Rhin passe par la Grèce, et la traduction qu'a entreprise Victor Cousin, en France, de tous les dialogues de Platon--à l'image de celle qu'a donnée Schleiermacher à l'Allemagne--est un enjeu stratégique décisif dans le champ agonistique de la philosophie européenne. Platon contre la philosophie du XVIIIeme siècle, parce qu'on y lirait que la raison est la source propre de toute connaissance. ${ }^{3}$ Mais aussi audessus de Kant, la première référence allemande de Cousin, parce que celui-ci mène au scepticisme.

Même si Hegel récrimine: "Kant tant au-dessous de Platon? Les Modernes au-dessous des Anciens? Sous beaucoup de rapports sans doute, mais pour l'étendue et la profondeur des principes? Nous sommes en général sur une ligne plus élevée." Un Hegel qui déjà s'était vivement ému de la sortie, un peu virulente envers le gouvernement auquel il est attaché, qu'il avait lue précisément dans l'argument du Gorgias, mais qu'il avait pardonnée à Cousin'; car Cousin, dans cet argument comme dans les autres, ne fait pas seulement dans l'érudition et dans l'histoire de la philosophie, mais actualise le sens et la portée philosophique de chaque dialogue, allant ici jusqu'à la politique de l'Etat prussien. De ces arguments, Jules Simon, qui fut le disciple de Cousin, son secrétaire et son suppléant dans sa chaire à la Faculté des lettres de Paris, écrit qu'ils sont plus qu'un guide pour le lecteur, à l'image des sommaires de Marsile Ficin, des notes marginales de Jean de Serres, des arguments en latin de Tiedemann, mais de belles introductions philosophiques qui

${ }^{3}$ Heinz Wismann. "Modus interpretandi", dans Philologic und Hermeneutik um 19. Jahrhundert, II, (Gottingen, 1983) et Patrice Vermeren, Platon communiste (Santiago du Chill, Cerc, 1987), repris dans les Cahiers de L'ENS Fontenay, 51/52 (septembre 1988): pp. 165-187.

- Lettre de Hegel a Cousin du 25 mars 1828.

${ }^{3}$ Cf la lettre de Carove a Cousin du 27 decembre 1826, dans laquelle celui-là disait deja attendre en vain l'arrivee promise, par une lettre du 4 aoot de celui-ci, de son ourrage. 
permettent de comprendre l'esprit du texte malgré sa lettre et de juger ses théories au présent ${ }^{6}$. Cousin a vu dans le Gorgias une réfutation de la fausse rhétorique, définie comme l'art de trouver des expédients mensongers pour paraître savoir, sans savoir en effet, aux yeux de gens qui ne savent pas; pour paraître juste, homme de bien, bon citoyen, sans l'être; enfin pour mettre partout l'apparence à la place de la réalité. Au contraire, la vraie rhétorique ne cherche pas à plaire ni à flatter, mais à être utile; elle aime et sert le peuple. Calliclès devient en conséquence celui qui réduit le philosophe au rhétoricien qui, connaissant son époque, marche à la domination par la parole et gouverne les hommes qu'il méprise, Socrate lui oppose son enthousiasme pour l'ordre légal et la justice, et les arguments les plus simples et les plus forts, tirés de la conscience du genre humain, et partout élevant le sens commun à la plus haute philosophie. Pour les lecteurs de 1826, la transcription à la situation politique du temps est immédiate, et comme si elle n'était pas assez explicite, Cousin en rajoute en plaçant en tête de son ouvrage la dédicace suivante:
A.M. G.W.F. Hegel, Professeur de Philosophie à l'Université de Berlin.

Je viens, mon cher Hegel, vous prier d'accepter l'hommage de cette traduction du Gorgias. Il était dû sans doute à celui qui le premier replaça avec honneur, parmi les principes éternels de la philosophie du droit, les maximes contenues dans cet antique monument.

Mais un autre motif encore me dirige en vous adressant cet hommage. Hegel, il $y$ a dix ans que vous me reçutes à Heidelberg comme un frère, et dès le premier moment nos âmes se comprirent et s'aimèrent. L'absence et le silence ne refroidirent pas votre amitié; et quand dans ces derniers temps, voyageant de nouveau en Allemagne, une police extravagante, dirigée à son insu par une politique

\footnotetext{
- Jules Simon. Oeurres completes de Platon traduites par V. Cousin, dans la Revue des Deux-Mondes, décembre 1840, p. 804-829.
} 
odieuse, osa attenter à ma liberté, me charger des accusations les plus atroces, et me déclarer d'avance convaincu et condamné, vous accourûtes spontané ment vous présenter devant me juges, leur dire que j'étais votre ami, et engager votre parole pour la mienne.

J'ai voulu, Hegel, vous remercier publiquement pour cette noble conduite, non pour vous ni pour moi, mais pour la philosophie. Vous avez prouvé qu'elle n'est pas toujours une occupation stérile, et que le génie de l'abstraction peut trés bien s'allier avec la fermeté de l'âme et le courage dans la vie. Encore une fois, Hegel, je vous en remercie.

\section{Victor Cousin \\ Paris, ce 15 juillet $1826^{7}$}

Gans, qui a reçu, contrairement à Carové, l'argument du Gorgias, a une autre version de sa réception par Hegel:

J'ai à peine eu le temps de lire votre courageuse et mâle dédication. Elle a fait beaucoup de joiè à Hegèl et il en a été tout à fait attendri. Que dira le baron de Humboldt, qui dans ce moment est a Berlin?

Juif converti par raison pour obtenir un poste à l'Université de Berlin (où Marx suivra bientôt ses cours), Gans est plus proche de Hegel et aussi de Cousin, qu'il a fréquenté à Paris après sa remise en liberté. Werner et Espagne citent dans l'introduction des Lettres

7 Deuvres de Platon, traduites par Victor Cousin. Tome troisieme. (Paris: Bossange freres, 1826): p. 3-4.

- Lettre a Cousin du 10 octobre 1826. 
d'Allemagne des extraits du livre que Gans publiera dix ans plus tard' et qui sont des indications précieuses sur l'état d'esprit dans lequel se trouve Cousin en 1826: il peut à la fois distinguer Robespierre et Saint-Just et louer M. de Villele; il est difficile de déterminer à quel parti politique il appartient au fond de lui-même; il est heureux de s'entretenir avec des Berlinois des souffrances de son incarcération, mais il défend aussi la monarchie prussienne:

\section{Déjà il n'était pas tout à fait étranger à l'idée} de venir en Prusse en mission extraordinaire et il ne voulait pas se gâter cette possibilité par une impétuosité qui ne lui aurait pas procuré le moindre avantage.

Sa destitution de l'Université en 1820 et son emprisonnement à Berlin en 1825 sous l'accusation de carbonarisme l'ont "sacré révolutionnaire malgré lui." 10 Lui qui n’a cessé, lors de ses interrogatoires comme à sa sortie de prison, de contester qu'il complotât avec des étudiants révolutionnaires, et de protester qu'il ne fit jamais de la politique qu'en philosophe défenseur de la Liberté, cherche à construire de lui-même une image très mesurée; à l'aune de ce que Hegel nomme le plomb impassible et irremuable de la malheureuse et inconcevable conduite que les allemands ont tenu envers lui. Puisque l'attente d'excuses officielles de la Prusse reste vaine, il n'y a plus qu'à rappeler l'injustice sans confirmer, par quelqu'audace, sa légitimité. Cousin peut publier, à l'intention de ses lecteurs français, une lecture de Platon qui prône la tolérance et donne l'ordre public comme un reflet de l'ordre moral, et rendre hommage au courage et à l'amitié de Hegel, affichant de ce fait qu'ils mènent tous deux, chacun sur sa propre rive du Rhin, un combat philosophique pour la vérité; et il peut envoyer à Gans-(-qui s'est rangé dans le clan des libéraux modérés et entre même en conver-

'Edouard Gans. Rackblicke auf Personen und Zustande. (Berlin: Veit, 1836). Cite par M. Espagne et M. Werner dans leur introduction.

10 Jules Simon. Victor Cousin. (Paris: Hachette, 1887): P.27; voir aussi article par Patrice Vermeren, "Les vacances de Cousin en Allemagne," dạns Raison Presente 63/64 (1982): p.123-132 et 101-119. 
sation avec Savigny, son rival de l'école historienne, pas du tout son ennemi-)-ou à Hegel, son argument du Gorgias; mais pas à ceux qu'un excès de tempérament révolutionnaire pourrait entraîner à en faire un usage déplacé.

Car tout est une question de place. Dans les lettres ici publiées se lit aussi la construction de la figure du correspondant autorisé: traducteur, commentateur, qui entre dans un système d'échanges réciproques complexe. Echanges de livres et de manuscrits, comme on l'a vu, selon une tactique sélective et discriminée. Echanges de bons offices pour la publicité. Par exemple, les allemands, de Goethe à Hegel, de Gans à Carové, sont tous séduits par le Globe qu'ils donnent comme paradigme du journal philosophique et politique du temps de la liberté. Ils cherchent à s'y faire publier, où à y faire rendre compte de leurs publications, et même, en Allemagne, à l'imiter. Cousin, qui y a des disciples-Jouffroy, Damiron, Barthélémy Saint-Hilaire--se garde bien de démentir publiquement sa proximité supposée avec une entreprise journalistique qu'il qualifie durement, pour ses audaces, en privé."1 S'il lui donne un article sur sa rencontre avec Goethe ${ }^{12}$, un texte sur l'histoire de la philosophie et quelques arguments des dialogues de Platon, il y apparaît plutôt sous la plume d'autrui à titre de philosophe respecté. Manière de conserver ce titre de Dieu tutélaire de tous les Allemands à Paris que lui décerne Gans pour lui recommander Mademoiselle Sontag, première cantatrice à la chambre du roi de Prusse, que celui-ci veut voir placée sous sa protection. En retour, on s'occupe de traduire les Fragments philosophiques en allemand, et de l'annoncer favorablement dans la Revue Encyclopédique, l'Allgemeine Litteraturzeitung et Le Protestant. Hegel au demèrant ne donnera jamais son article promis qui ne pouvait être remplacé--échanges de reconnaissances qui requièrent de dépasser le cercle confidentiel de l'amitié privée.

1 Voir Sainte-Beuve. "Jouffroy", dans la Revue des Deur-Mondes (1er decembre 1833), repris dans les Portraits litteraires (Paris: Garnier, Nouvelle Edition 1924), pp. 297-324.

12 Victor Cousin. "Visite à Goethe." dans Le Globe (2 juin 1827). 
Celle-ci paraît pourtant acquise à Cousin, qui en a reçu le témoignage direct lorsque Hegel intervint auprès du ministre prussien de l'intérieur et de la police pour le faire sortir de prison, et aussi lorsqu'il se réjouit de la nomination de Royer-Collard, un professeur de philosophie à la tête de la chambre, dont la composition a si furieusement trompé les calculs des gens en place, mais qui n'est pas encore prête à rétablir le cours suspendu de son ami. Pour saisir les enjeux de cette relation priviligiée, on possède un récit tardif de Cousin, écrit alors que la philosophie française s'est voulue radicalement émancipée de la métaphysique allemande, et que son père fondateur, attaqué pour l'avoir compromise avec la politique, tente une dernière fois de re-façonner la légende de ses origines. Où l'on retrouve Carové, dans un article publié dans La Revue des Deux-Mondes du Ier août sous le titre: "Souvenirs d'Allemagne":

Je suivis à peu près la même conduite avec M. Hegel (qu'avec M. Creuzer, au cours de mon voyage en Allemagne en 1817); j'essayais de l'entendre plutôt que de le juger. Il venait de publier son Encyclopédie des sciences philosophiques à l'usage de ceux qui fréquentaient ses cours. Je me jetais avidement sur ce livre; mais il résista à tous mes efforts et je n'y vis qu'une masse compacte et serrée d'abstractions et de formules bien autrement difficiles à pénétrer que les traités les plus hérissés de la philosophie scolastique. Les ouvrages de Saint-Thomas et de Duns Scott sont des badinages en regard de celui-là. Heureusement je rencontrais chez Hegel un étudiant de mon âge, jeune homme instruit et aimable, M. Carové, né à Trêves, et qui, déjà pourvu en son pays d'une petite place de judicature, l'avait quittée pour venir écouter M. Hegel à Heidelberg, s'attacha à sa fortune, le suivit a Berlin et fut quelque temps privat-docent et même, je crois, professeur extraordinaire à l'Université de Breslau. Cest lui qui a bien voulu traduire en allemand mes premiers essais, et dans cet automne de 1817, il me rendit le service de lire avec moi quelques chapîtres de la terrible Encyclopédie. Plusieurs fois par semaine, nous nous réunissions le matin, et à travers 
les ruines du vieux château, ou par ce sentier charmant que tout le monde connaît à Heidelberg sous le nom de "Sentier des Philosophes," nous nous promenions, le manuel de Hegel à la main, moi lui adressant des questions, lui me répondant avec une complaisance infatigable; mais en vérité le jeune maître n'était guère plus. avancé que son écolier, mes questions restaient souvent sans réponse, et le soir nous allions ensemble prendre le thé chez M. Hegel, à la manière allemande, et interroger l'oracle, qui luimême ne m'était pas toujours fort intelligible (...). Ainsi décidé à revenir bientôt à Heidelberg, je me bormais à y prendre cette fois un simple avant-goût de cette doctrine qui devait faire un jour tant de bruit en Allemagne. ${ }^{13}$

Cousin prétend avoir été prévenu, dès ses premiers contacts avec Hegel, contre l'obscurité de son système. Il ajoute que l'ordre ternaire inflexible de sa dialectique appliqué en tout éveilla ses premiers ombrages et lui rendit suspect le système entier. Il ne reconnaît en 1866 qu'une seule proximité avec Hegel et qui a perduré:

En politique, M. Hegel est le seul homme d'Allemagne avec lequel je me suis toujours le mieux entendu. Il était, comme moi, pénétré de l'esprit nouveau; il considérait la Révolution française comme le plus grand pas en avant qu'eut fait le genre humain depuis le christianisme, et il ne cessait de m'interroger sur les choses et les hommes de cette grande époque. Il était profondément libéral sans être le moins du monde républicain. Ainsi que moi, il regardait la république comme ayant été nécessaire pour jeter bas l'ancienne societe, mais incapable de servir à l'établissement de la nouvelle, et il ne séparait

13 Victor Cousin. "Souvenirs d'Allemagne." Revue des Deur-Mondes, Ier aont 1866, p. 66. 
pas la liberté de la royauté. Il était donc sincèrement constitutionnel et ouvertement déclaré pour la cause que soutenait et représentait en France RoyerCollard.

Ce témoignage reconstruit l'histoire, mais en propose aussi une certaine vérité. Le hégelianisme philosophique de Cousin n'a jamais été par lui affiché, et dans ses cours de 1828 , où l'influence du maître de Berlin ne peut être niée, jamais son nom n'est prononcé. Hegel, on le sait, en aurait pris ombrage, et dit que Cousin lui avait pris quelques poissons, mais les avait noyés dans sa sauce. Mais la connivence politique, par la dédicace de 1826, était aux yeux de tous proclamée.

Hegel lui-même avait écrit à Cousin, le Ier juillet 1827:

"Cette dédicace, monument de vos sentimens amicales pour moi, contient enfin votre manifeste contre notre police, pour l'omniscience de laquelle au reste Platon est vraisemblablement un coin obscur, dans lequel probablement elle n'a pas pénétrél"t".

Mais l'amitié, même confortée par le partage d'opinions politiques, ne peut aller jusqu'à masquer les différences philosophiques. C'est ainsi que Hegel explique en 1830 son silence sur les publications de Cousin, alors qu'il se croyait dans l'obligation de lui adresser une grosse épître devant le public: le philosophe français a parlé trop, ou trop peu, de l'histoire de la philosophie allemande, et singulièrement de Schelling, et le philosophe allemand ne voulait pas rendre manifeste aux yeux du monde philosophique cette réticence. Comme par ironie du sort, lorsqu'en 1831, Hegel meurt, la nécrologie que rédige Gans est censurée par la Gazette d'Etat; on n'y trouve plus trace de sa liaison avec Cousin.

Après 1830, la correspondance de Cousin avec les hégeliens atteste moins de préoccupations philosophiques et culturelles que de soucis proprement institutionnels. Victor Cousin est devenu membre

${ }^{14}$ Lettre de Hegel a Cousin du Ier juillet 1827 , republiee par M.E. et M.W. 
du conseil supérieur de l'instruction publique, pair de France, chef de la section philosophie de l'Académie des sciences morales et politiques, président de l'agrégation; il se fait suppléer dans sa chaire et n'enseigne plus. Il retourne en Allemagne pour enquêter sur l'instruction publique, et s'arrête â Francfort sans saluer Carové, qui en reste fâché. Recevant finalement la seconde édition des Fragments, Carové lui écrit le 26 décembre 1833:

Cependant celà ne m'a pas empêché de Vous suivre dans votre carrière politique et de me réjouir de tout le bien que Vous faites, et du succès dont Vos travaux sont couronnés. J'ai été bien aise de Vous voir prendre place dans le sénat de la nouvelle république française, car il est tems, ce me semble, que la vraie philosophie devienne pratique et que les hommes de bons et hauts principes deviennent hommes d'action. Les penseurs éminens se trouvent nantis d'un trésor d'idées qu'il est tems de répandre dans la réalité et de faire fructifier, en attendant des inspirations encore plus complètes. 15

De plus en plus désormais, la correspondance porte sur des nominations à des postes universitaires, des élections de correspondants étrangers dans les Académies, des prix de concours. La stratégie de Cousin vise à conforter sa position de "médiateur entre les modes de pensée philosophique et les aspirations des deux nations et qui occupe parmi les philosophes de l'une d'entre elles une position aussi distinguée et glorieuse." ${ }^{16}$ Lorsque Charles Michelet remporte le prix du concours de philosophie de l'Académie des sciences morales et politique, qu'il partage avec Ravaisson, sur la Métaphysique d'Aristote, il crédite Cousin de son propre succès: "Cest vous qui le premier avez attiré sur la philosophie allemande l'attention de la France, et procuré par là à cette philosophie un nom européen." Il lui demande de s'entremettre pour publier son livre à Paris, parce que c'est la meilleure façon d'y intéresser les allemands,

${ }^{15}$ Lettre de Carove a Cousin du 23 decembre 1833, ibidem.

${ }^{16}$ Lettre de Weisse a Cousin du 18 mai 1835, ibidem. 
puis une chaire à la Sorbonne ou au Collège de France. Mais le chaînon qui relie la philosophie française à la philosophie allemande préfere sans doute garder à distance ce concurrent et lui faire jouer un rôle d'intermédiaire à Berlin, et d'informateur sur la guerre déclarée entre les hégeliens, Schelling et les derniers kantiens. Lorsque Cousin est nommé ministre, un Forster n'hésite pas à célébrer l'évènement comme une victoire de la philosophie, et à tenter de placer son futur gendre à un poste de professeur ou de bibliothécaire. Et Carové, cinq ans plus tard, lui demande de rapporter l'engagement dans la légion étrangère du fils d'une amie. Au demeurant, le choix de lettres que Werner et Espagne publie ne retient pas toutes les lettres de sollicitation que l'on peut lire dans les archives de la Bibliothèque Victor Cousin. Il conserve les correspondances qui décrivent les enjeux proprement intellectuels de l'introducteur de la philosophie hégelienne en France avec les derniers représentants de ce parti en Allemagne.

On en tirera deux considérations. D'abord que le sol grec de référence des travaux philosophiques franco-allemands se déplace de Platon à Aristote, aux alentours des années 1840. Faut-il y voir Pinfluence de Schelling? Sans doute. Mais aussi le souci de se rapprocher de l'expérience et de la légitimation du pouvoir des capacités, quand l'idéalisme platonicien, dans l'appropriation que s'en font les utopistes et les prolétaires, est revendiqué comme paternité des rêves d'émancipation du peuple. Il est intéressant de remarquer de ce point de vue que la dernière traduction de Platon précédée d'un argument dont il soit question est celle des Lois, et que la République paraîtra sans appendice cousinien.

Ensuite, que Cousin a finalement explicité en 1849, ses distances prises depuis longtemps avec le hégelianisme, une lettre de Charles Michelet en décrit longuement l'argumentation, qui procède de l'idée générique que la philosophie hégelienne a causé tous les maux dont l'Allemagne et la France sont maintenant atteintes: athéisme, panthéisme, républicanisme. Retour à la philosophie française et au sens commun, haro sur Hegel, dont le dernier rejeton en France, pour avoir franchement avoué qu'il ne croyait ni en Dieu, ni au diable, n'est autre, selon Cousin, que Proudhon. 
Cet ordre d'argumentation signe l'éviction durable de la référence hégelienne dans l'institution philosophique française. Le dernier signe en est la suspension du directeur des études à l'école normale supérieure, Etienne Vacherot, en 1851, après la réfutation par l'aumônier de l'établissement de son ouvrage sur l'école d'Alexandrie. Barbey d'Aurévilly incrimine en ces termes le professeur de philosophie pour son allégeance à Hegel:

"(Vacherot), avec son principe d'identité, n'a-t-il pas soutenu qu'on peut affirmer le pour et le contre, d'un même sujet, dans le même sens et sous le même rapport? Cest la même formule, popularisée par Proudhon, de la thèse, de l'antithèse, et de la synthèse. Or on comprend qu'en vertu de ce système, auquel $M$. Vacherot a donné une publique adhésion, les contradictions se pressent dans son livre; on comprend quelles énormités doit produire ce système même dans un esprit mou, comme celui de $M$. Vacherot. A cette heure, l'Allemagne philosophique qui l'appliqua en grand, ce système, offre à l'observateur un prodigieux et effrayant spectacle. J'ose le dire, si cette philosophie, dont $M$. Vacherot admire la grandeur et la fécondité, continue de se propager encore quelques temps en Allemagne, elle y défoncera la tête humaine, et l'esprit tarira dans la patrie de Goethe, comme le sang tarit dans une veine rompue!"

Et Barbey d'Aurevilly de citer Ewerbeck a titre d'épouvantail théorique, soit un disciple de Hegel, médecin ami de Grun et de Mòses Hess qui vire bientôt au proudonisme, après avoir traduit le Voyage en Icarie de Cabet en allemand:

Je le répète, je ne le répèterai jamais assez: voila la philosophie allemande que $M$. Vacherot, dans son

${ }^{17}$ Ewerbeck. Qu'est-ce que la religion d'apres la nouvelle philosophie allemande? (1850); Barbey d'Aurevilly: "Lettre a M. Vacherot, directeur des études a l'ecole normale, par l'abbe Gratry, aumonier de l'école normale", paru dans l'Assemblée Nationale du 4 août 1851. 
livre, vante comme possédant la solution du problème de la vérité. A part les erreurs, les contradictions, les ignorances, quand elles ne sont pas des mensonges, de l'ouvrage de $M$. Vacherot, il n'y aurait que l'éloge, l'admiration, le respect du chef de la première école française pour une philosophie pareille, que le gouvernement devrait intervenir et arracher ce chef d'école à l'enseignement devenu un empoisonnement entre ses mains.

Le journaliste catholique ne dit ici rien d'autre que ce que son parti a répété depuis plus de quinze ans; et l'on comprend pourquoi Cousin attendra le soir de sa vie pour publier le récit de ses premières rencontres avec Hegel dans les termes que l'on sait (voir supra), tandis qu'il cherchait à éviter la mise à l'index par Rome de son $D u$ vrai, du beau, du bien. ${ }^{18}$

Cette correspondance entre Cousin et Hegel et ses disciples allemands, d'un intérêt considérable, peut être l'objet de plusieurs lectures. Jules Barthélémy Saint-Hilaire, disciple très orthodoxe de Victor Cousin, en tirait argument pour l'hagiographie militante de son maître, tout comme Paul Janet ${ }^{19}$. L'introduction de la philosophie allemande en France est soumise a un impératif constant, la proportionner au tempérament français, la faire servir à un renouveau de notre philosophie nationale. Jacques Derrida en propose un bel usage philosophique contemporain, du côté de la revendication polémique d'un enseignement philosophique appliqué aux enfants ${ }^{20}$

18 Voir Amine A. Azar. "Le cas Victor Cousin: un étrange observateur de la pensée germanique pendant le début du XIXeme siècle", dans Critique 473 (octobre 1986).

19 Paul Janet. Victor Cousin et son oeuvre. (Paris: Félix Alcan, 1893): p. 180-214 and Jules Barthelemy Saint-Hilaire. Victor Cousin, sa vie et sa correspondance. (Paris: Hachette/Alcan, 1895): pp.79, 80, 128, 151, 214, 227, 379 du tome I.

20 Jacques Derrida. "L'age de Hegel." dans Qui a peur de la philosophie? (Paris: Flammarion, 1979): pp. 73-107. 
ou de celui d'une définition de la philia ${ }^{21}$. Michel Espagne et Michael Werner sont soucieux d'en tirer un paradigme des transferts culturels franco-allemands; Cousin aurait utilisé le hégelianisme moins dans l'ordre du philosophique que dans celui du politique, pour légitimer sa politique institutionnnelle de fondation de l'Universitéén. Il faut cependant, pour en prendre toute la mesure, confronter cette correspondance avec celle que Cousin entretient avec Schelling, qui atteste, dans la virulence de ses raisons agonistiques, que la circulation et la diffusion en France de la pensée allemande est aussi un enjeu métaphysique ${ }^{23}$, et la mesurer à l'aune des autres usages de la naturalisation de la pensée allemande en france, par exemple chez Quinet ou les saint-simoniens ${ }^{24}$. Le travail de Werner et Espagne est désormais incontournable pour ceux qui veulent construire aujourd'hui un concept de l'identité philosophique européenne.

Patrice Vermeren

Centre National de Recherche Scientifique

21 Jacques Derrida. Glas. ( Paris: Galilee, 1974).

2 M. Espagne, et M. Werner. "Transferts culturels franco-allemands." Revie de Synthèse 2 (avril-juin 1988): p. 17.

${ }^{23}$ Christiane Mauve et Patrice Vermeren. "Le passage de la ligne: Politique de la nationalite philosophique sur les deux rives du Rhin", suivi du "Jugement de Schelling sur la philosophie de M. Cousin et sur l'Etat de la philosophie française et de la philosophie allemande en general" (1833) dans les Cahiers du College international de philosophie 6 (octobre 1988): pp. 53-84 ; Dominique Janicaud, "Victor Cousin et Ravaisson, lecteurs de Hegel et de Schelling," dans Les Etudes Philosophiques 4 (octobre-décembre 1984): pp. 451-466. Voir aussi le numéro special de la revue Corpus consacre a Victor Cousin, à paraftre en janvier 1992.

24 Pierre Penisson. "Michelet, Quinet et l'Allemagne" dans la Revue de synthese 2 (avril-juin 1988): pp. 247-263; Michel Espagne. "Le saint-simonisme est-il jeune hégelien?" dans Regards sur le saint-simonisme et les saint-simoniens. (Lyon: Presses universitaires de Lyon, 1986): pp. 45-71. Miguel Abensour, "Pierre Leroux ou du 'style barbare'en philosophie," dans tes Cahiers du College international de philosophie 1 (November 1985): pp. 9-24. Voir aussi la these de Georges Navet. De l'usage de Vico en France: le problème de la legitimite du droit civil. (Reims: Universite de Reims, 1987), et Jacques d' Hondt. "Hegel et les socialistes", dans La Pensee 157 (juin 1971): pp. 1-25; Hegel en son temps (Paris: Editions sociales, 1968), et "Le probleme du passage a l'acte politique", dans Philosophique, revue de l'Universite de FrancheComte, 1988, 1, pp. 553-74. . 